$\begin{array}{ll}\text { le portiQue } & \text { Le Portique } \\ \text { Revue de philosophie et de sciences humaines }\end{array}$

$11 \mid 2003$

\title{
Le Respect
}

\section{Faut-il respecter le corps humain?}

\section{Bernard Andrieu}

\section{OpenEdition}

\section{Journals}

Édition électronique

URL : http://journals.openedition.org/leportique/553

DOI : $10.4000 /$ leportique.553

ISSN : $1777-5280$

\section{Éditeur}

Association "Les Amis du Portique"

Édition imprimée

Date de publication : 1 janvier 2003

ISSN : 1283-8594

\section{Référence électronique}

Bernard Andrieu, «Faut-il respecter le corps humain ? », Le Portique [En ligne], 11 | 2003, mis en ligne le 15 décembre 2005, consulté le 25 mars 2021. URL : http://journals.openedition.org/leportique/553 ;

DOI : https://doi.org/10.4000/leportique.553

Ce document a été généré automatiquement le 25 mars 2021

Tous droits réservés 


\title{
Faut-il respecter le corps humain?
}

\author{
Bernard Andrieu
}

Introduction : Du respect de l'autre à la guerre du soi

1 Le respect du corps est devenu une norme juridique, une obligation morale, un critère de dignité ou encore une formule de politesse. Contre la violence, le respect à l'école devrait suffire pour restaurer la hiérarchie sociale. L'intégrité corporelle est réifiée au rang d'exigence d'interpersonnelle, renforçant le regard comme agent communicationnel et interdisant toute forme de toucher. Être touché sans y consentir, physiquement et moralement, est devenu le critère de l'irrespect, car en touchant le corps, on porterait atteinte à la personne. Manquer de respect est un symptôme de la crise identitaire: faute d'une estime de soi suffisante, susceptible de résister au jugement d'autrui, le moi contemporain perdrait ses composants identitaires par la mainmise sur son corps. Conserver son corps entier définit un intégrisme fonctionnel, si bien que toute dégradation de l'unité physique et mentale est ressentie comme une perte. L'haptophobie maintient les autres à distance dans des systèmes de comparaisons. L'autre est tenu en respect, condition de mon respect pour lui.

2 Kant avait su, dès les Fondements et plus sûrement dans la seconde Critique, définir le respect comme une humiliation de la naturalité par la moralité ; la sensibilité qui nous fait agir conformément au devoir plutôt que par devoir pourrait nous détourner de la dimension universelle du respect. La guerre peut-elle être une simple continuation d'une politique du respect en imposant la force contre le droit? La guerre est bien un acte politique où la force entre en ligne de compte. Trois discours structurent l'analyse de la guerre :

3 - Le discours naturaliste selon lequel la force serait un élément naturel et la guerre une dérive naturelle de la chasse. Thèse du droit naturel avec spécialisation d'un groupe social.

4 - Le discours économiste pour lequel la rareté, c'est-à-dire la compétition autour des ressources, serait à l'origine de la dispute entre les hommes. La critique de cette thèse aura été de faire remarquer que, contrairement à l'opinion selon laquelle les sociétés dites primitives seraient des sociétés de la rareté, elles vivent en abondance par rapport à la définition des besoins en rapport avec le milieu. 
5 - Le discours échangiste analyse la guerre comme un échec dans l'échange des individus, des biens ou des signes dans la mesure où une réciprocité aurait été interrompue.

6 La guerre transforme la lutte intérieure entre la naturalité et la moralité en une imposition par la force de ce qu'il faudrait respecter. En cela la guerre est une condition contemporaine du sujet au respect : au lieu de s'y soumettre par la contrainte morale, le sujet veut tout soumettre par la force physique.

7 La nature, l'homme, le corps ne sont ainsi plus l'objet de respect. Non seulement leurs transformations économiques en marchandises, mais leurs modifications génétiques prouvent l'immanence de la Nature. En se libérant de la nature, le sujet occidental remplace le respect de son origine par le respect de lui-même, supprimant ainsi toute transcendance entre le sujet qui respecte et l'objet respecté ; le corps est réifié au rang de matière à subjectiver, à rendre digne de respect, de l'idée que l'on se fait de soimême. Ces jeunes ne respectent plus rien, car rien du monde extérieur aliéné ne correspond à ce qu'ils désirent ${ }^{1}$. L'irrespect est moins une incivilité qu'une survalorisation identitaire du respect que l'on accorde à soi-même: manquer de respect serait une atteinte à la dignité, à l'image de soi-même et une négation de son identité.

8 Le soi fait la guerre aux autres, là où le respect des autres faisait la guerre à l'égoïsme. Mais dès lors que l'espoir est dans mon corps ${ }^{2}$, plutôt que dans le corps de l'autre, devenir soi-même plutôt qu'admirer des modèles fait croire à chacun qu'il est digne de respect. Ou plutôt que le respect vaut en lui-même, ne repose sur aucune intersubjectivité. L'auto-chirurgie du sens ${ }^{3}$ trouve dans le corps le moyen de définir une identité mobile, flexible et recomposable. Le respect de soi comme identité inflexible et substance permanente n'est plus suffisant pour donner au sujet moderne le sentiment d'une dignité construite plutôt que reçue.

La Maîtrise de son corps comme l'invention du respect de soi

9 David Cronenberg souligne, depuis Rage, Videodrome, La Mouche, Chromosome III, eXistenZ, combien la sexualité est sortie de la reproduction naturelle pour devenir un jeu: le respect se conquiert dans l'épreuve, le rôle, le mélange et le jeu sans que s'impose désormais le déterminisme des sexes et les nécessités de la nature. La maîtrise des mécanismes naturels permet de choisir son corps ${ }^{4}$, obligeant le sujet à redéfinir le respect comme une valeur immanente et non plus transcendante à soi-même. Il faut d'abord mettre en avant la verticalité de la filiation en liant le corps naturel et fécond avec la formation de la famille : la famille élémentaire se compose d'un homme et d'une femme et d'enfant(s) dont la production est due à la sexualité fécondante. Ce fondement biologique de la famille ramène toutes les autres formes de lien social à une relation corporelle sexuée: dans la mesure où le tabou de l'inceste ne prend de signification qu'entre relations de parenté sans dimension symbolique susceptible de surdéterminer la relation biologique du corps des parents à celui des enfants. $\mathrm{Ce}$ modèle assure une continuité du temps dans l'enchaînement de la trame familiale des ascendants et des descendants. La verticalité ne retient du corps humain que sa dimension biologique et fécondante. Une telle définition ne pouvait qu'être profondément remise en cause dès lors que la chimie des hormones a permis, à partir de 1956-1957, après les travaux de Gregory Pincus à Porto Rico, de contrôler l'ovulation par la maîtrise de la progestérone. En effet, la famille ne sera plus le résultat d'une 
relation naturelle d'un corps masculin avec un corps féminin, mais le résultat d'un choix.

Dès la loi du 31 juillet 1920, la répression est organisée contre toute provocation ou incitation à l'avortement ou à la propagande anticonceptionnelle. La natalité reste la conséquence naturelle de la sexualité. Par la suite, le Code de la famille du 29 juillet 1939 avait encore renforcé certaines dispositions précédentes et le décret du 5 février 1946, relatif aux moyens anticonceptionnels, apportait des précisons: ainsi l'article premier de ce dernier décret énumérait les substances qui pouvaient être délivrées "seulement sur prescription médicale, et transcrites sur un registre coté et paraphé par le maire ou le commissaire de police ». La prescription médicale restera une constante, même lors de la présentation officielle de la première pilule en 1957: l'organisme américain de contrôle des médicaments autorisa l'utilisation de l'Enovid d'abord uniquement comme régulateur de menstruation. Puis, en 1960, sa prescription comme contraceptif oral reçut le feu vert avec une restriction : le traitement ne devait pas dépasser deux ans. Cette dimension médicale, outre les précautions de prescription, s'explique aussi sans doute par la protection de la famille. Car la pilule ne venait qu'en cas de situation exceptionnelle dans les premières prescriptions, en cas de dysfonctionnement du corps naturel dans son cycle biologique.

11 Il faut percevoir le combat féministe de Margaret Sanger pour comprendre la perspective sociale de la maitrise de l'ovulation par la chimie des hormones. Née en 1879 dans une famille de onze enfants, poussée par Emma Goldman une militante féministe, Margaret Sanger lança en mars 1914 le premier numéro d'une revue intitulée Femme en révolte. Ni dieux, ni maîtres. L'objectif est de rendre aux femmes la maîtrise de leur vie et de leur corps qui passait par la liberté de refuser les grossesses non désirées. Condamnée, elle fuit à Londres où elle rencontre Havelock Ellis, pionnier de la psychologie de la sexualité. De retour aux États-Unis, elle fonda en 1923 le Bureau de recherches cliniques sur le contrôle des naissances: sa mission est d'informer les femmes sur les méthodes contraceptives disponibles: diaphragmes et spermicides, douche et préservatif. En 1926, le centre de Margaret Sanger avait déjà reçu plus d'un million de lettres provenant de femmes intéressées par la contraception. 250 centres de planning familial avaient été ouverts dans 150 villes américaines. En 1913, elle importa d'Europe le diaphragme, interdit aux États-Unis, puis en 1930, les crèmes spermicides d'Allemagne.

12 En se libérant du déterminisme naturel, la femme a modifié son identité sociale en ne devenant plus nécessairement mère : la famille n'indiquait pas la conclusion biologique de la relation sociale. Elle prend pour essor le moyen de la contraception pour définir la volonté de filiation comme la conséquence d'un choix. La famille ne devient plus une obligation naturelle, même si le regard social pèse sur la femme mariée ou célibataire. Prendre en compte ce choix modifie l'action sociale avec l'apparition du planning familial. En 1956, Évelyne Sullerot crée avec le Dr Andrée Lagroua-Weill-Hallé l'association Maternité heureuse, qui devient en 1958 le Mouvement français pour le planning familial. Dès 1961, le Planning familial ouvre deux centres de consultation à Grenoble et à Paris: les militantes féministes commencent par distribuer des diaphragmes importés d'Angleterre ou de Suisse. En 1966, le Planning compte 42 centres et 37 permanences dans 57 départements. Visant à abroger la loi sur le contrôle des naissances de 1920, le gaulliste Lucien Neuwirth obtient, en 1967, une majorité de socialistes, communistes et de la droite libérale pour le 28 décembre voter la loi 
$\mathrm{n}^{\circ}$ 67-1176. Elle autorise l'accès aux méthodes contraceptives, leur fabrication et leur importation. Les contraceptifs seront distribués sur ordonnance, laquelle s'accompagnera d'un bon tiré d'un carnet à souches permettant un contrôle du médecin. les stérilets ne pourront être posés qu'à l'hôpital. Pour les mineurs, c'est-àdire à l'époque les moins de 21 ans, le consentement de l'un des parents est requis. La structure familiale semble protégée dans la mesure où l'autorisation parentale est paradoxalement nécessaire pour éviter aux enfants de devenir eux-mêmes parents par déterminisme naturel. Jusqu'en 1971, la pilule n'est quasiment distribuée que dans des centres du Planning familial. Il faudra attendre 1972 pour que les décrets d'application soient publiés au journal officiel.

13 Au même moment a lieu le procès de Bobigny ${ }^{5}$, publié par l'association Choisir. L'association a été fondée par Simone de Beauvoir, Gisèle Halimi, Christiane Rochefort, Jean Rostand, Delphine Seyrig et reçoit le soutien de Jacques Monod et François Jacob. Les objectifs de l'association sont :

14 «1) Rendre la contraception libre, totale et gratuite.

15 2) Obtenir le suppression de tous les textes répressifs relatifs à l'avortement.

16 3) Défendre gratuitement et assister toute personne accusée d'avortement ou de complicité ».

17 Une mère célibataire de trois filles aide l'une d'entre elle à se faire avorter; elle est jugée devant le tribunal de Bobigny qui la condamne à 500 francs d'amende avec sursis. En 1972, le chiffre officiel est de 400.000 avortements alors que 800.000 sont pratiqués, dont 500 sont condamnés en moyenne. De 1965 à 1970, on passe de 588 à 340 condamnations. Au terme du livre, on peut lire une proposition de loi relative à l'interruption de grossesse présentée par les députés Michel Rocard, Aimé Césaire, Louis Vallon et David Rousset ; on peut lire dans ce texte le passage suivant :

«Héritage du Code de la Famille (29 juillet 1939), cette répression connut son paroxysme devant les juridictions pétainistes où une femme ayant pratiqué des avortements fut condamnée à mort et exécutée... la loi répressive ne sévit que pour sanctionner les femmes et les hommes les plus vulnérables, économiquement et socialement parlant. L'étude systématique des condamnations prononcées ces dix dernières années illustre... le caractère d'une justice de classes. C'est en effet parmi les couches les plus défavorisés de la population que l'avortement clandestin est vécu comme un drame: par les circonstances de l'intervention, souvent désastreuses pour la santé physique et psychique de la femme, par le coût prohibitif des services des avorteurs hors-la-loi, par la dénonciation policière, et enfin par le procès devant les tribunaux, humiliant et inique ". L'avortement n'est pas cependant présenté par les rapporteurs comme un moyen de réguler les naissances. Invoquant la courbe de natalité de l'URSS entre 1913 et 1963, ils concluent que celle-ci ne s'est pas modifiée entre les périodes de libéralisation de l'avortement(1913-1927 et 1955-1963) et de prohibition (1937-1955).

C'est seulement en 1974 que Simone Veil, ministre de la Santé de Valéry Giscard D'Estaing, fait voter le 4 décembre la loi 74-1026 avec les décrets d'application au 17 janvier 1975 : elle fait disparaître des textes le carnet à souches, l'autorisation parentale pour les mineures, et introduit le remboursement par la Sécurité Sociale des contraceptifs. Sur les 291 représentants de la majorité de droite, 99 votèrent seulement pour la loi, qui, en définitive, ne fut adoptée que grâce à la quasi-totalité des voix de l'opposition socialiste et communiste. Pourtant, l'avortement et la libéralisation de la contraception n'ont pas remis en cause profondément la structure familiale. Il faut examiner tous les facteurs : la modification de la structure familiale n'est pas seulement 
due à l'avènement de la contraception et du divorce. Une profonde modification sociologique et idéologique est apparue dans l'après 1968 en attribuant aux femmes un facteur de mobilité et d'investissement social : dans le travail, dans la sexualité, dans la reconnaissance idéologique juridique et morale des combats du féminisme. La crise économique des années 1975 a aussi modifié la dimension de l'engagement temporel par la possibilité ou non de se projeter à travers la fécondité dans l'avenir.

Aujourd'hui, un pas supplémentaire est franchi. Dans son Manifeste contra-sexuel, Beatriz Preciado dénature l'hétérosexualité en construisant une théorie du corps qui se situe en dehors des oppositions: «la contra-sexualité affirme qu'au commencement était le gode ${ }^{6}$, La nature ne doit plus apparaître tant dans sa forme, sa matière, sa différenciation comme un principe digne de respect ; le gode, objet technosexuel stérile par excellence, achèverait la libération sexuelle en accordant non plus seulement une maitrise contraceptive mais une auto-sexualité homosexuelle dans laquelle l'altérité serait entièrement culturelle et sociale. "La boîte de Pandore Lesboqueer $~^{7}$ définitelle des Queer zones ${ }^{8}$ si différentes de l'opposition respect-irrespect en faisant de la transgression un mode de régulation sociale face au pouvoir normatif de l'idéologie? Comment décrire un corps à soi qui critique l'interprétation masochiste et soit ouverture à un érotisme tactile d'autrui ${ }^{9}$ afin d'éviter d'exalter la vue et le visible comme un système panoptique de respect?

La recherche quantitative d'un hédonisme individualiste réduit le respect d'autrui à son instrumentalisation ${ }^{10}$. Sade, dans son œuvre et dans sa vie, refuse toute action sur la Nature. Aujourd'hui, l'espèce humaine veut en finir avec la Nature en la remplaçant par un environnement artificiel. La domination de la nature fournit l'illusion de son élimination. Les progrès de la technique font accroire au sujet humain que la Nature peut être transformée en environnement. Toute la globalisation de la planète vient troubler, par des intempéries climatiques et la destruction des écosystèmes, notre maîtrise. La Nature ne se rend pas, elle résisterait à sa mise en conformité technologique. Sade maintenait la Nature face à l'homme mais aussi en l'homme: la maîtrise de soi et le développement personnel se fondaient dans des rites de soumission et d'initiation. En devenant naturel, le sujet sadien devait retrouver sa véritable nature : l'harmonie ne pouvait être possible que si le sujet ne prétendait plus résister à ses instincts.

21 Le combat humaniste contre cette définition naturelle de l'homme a cru pouvoir proposer la culture comme un rempart au sadisme. Pourtant, la question du mal, si illustrée au cours $\mathrm{du} \mathrm{xx}^{\mathrm{e}}$ siècle par les crimes contre l'humanité, découvrait la perversité au cœur de l'homme. L'échec de la culture des Lumières à éclairer le jugement et l'action des criminels du génocide interroge le caractère grave du crime. Pour Sade, le seul crime réel est de résister à la Nature. Mais s'abandonner à la Nature, comme P. P. Pasolini a pu le représenter dans son film Salo ou les Cent-vingt Jours de Sodome, revient à livrer toute société au droit du plus fort: la puissance naturelle de dominer ses semblables les assujettit sans les libérer volontairement des contraintes culturelles.

Sade interroge notre idéologie de la libération de soi en nous proposant de vivre sans limites naturelles et sans règles culturelles. Le droit à disposer de son propre corps serait la définition universelle du respect de la personne humaine. Pourtant, le droit à disposer du corps d'autrui par son exploitation laborieuse ou par sa sélection génétique entame le respect universel de l'humanité. Comme le prévoyait Sade, la recherche du 
plaisir individuel sert désormais d'alibi au recul indéfini des limites. Aucune valeur culturelle ne serait désormais suffisante pour interdire la réalisation de notre désir.

L'intention eugéniste contre l'humiliation de l'individualité par l'universalité

La stérilisation des handicapées mentales en France ${ }^{11}$, mais aussi l'élimination du handicap diagnostiqué comme virtuel ou possible sont des techniques de bio-pouvoir qui redéfinissent le respect du corps de l'autre. La liberté de choisir son corps naturel est nulle et, une fois née, la personne humaine pourrait, à condition d'y avoir accès, modifier l'être reçu par une médecine réparatrice. Chacun veut non seulement être médecin de son corps ${ }^{12}$ mais désormais du corps de l'autre, en incorporant le respect dans la forme matérielle de l'enfant à naître afin qu'il ait une existence digne. Il manifesterait, pour autant qu'il en ait conscience (ce qui n'est pas toujours le cas pour les enfants sous tutelle et/ou déficients mentalement), l'intention de se libérer comme sujet de cet état naturel, donnant ainsi son consentement explicite. En agissant sur le corps à naître dès sa conception, l'intention eugéniste, selon Habermas ${ }^{13}$, interdirait et condamnerait toute subjectivité à la dépendance parentale du géniteur. La qualité du corps vivant déterminerait le corps vécu, l'image du corps, la conscience intergénérationnelle et la relation filiale. L'intention eugénique, à travers la reprogrammation et la sélection du génome, précède le sujet, ce qui l'empêcherait de devenir un être intentionnel à part entière: son corps déciderait de son degré d'intentionnalité et de communicabilité, son être serait amoindri. Cette détermination du corps vécu par l'intentionnalité sanitaire du corps vivant présuppose que le sujet préexiste à la qualité de son corps. L'évitement de l'instrumentalisation génétique du corps humain garantirait l'exercice de la subjectivité en maintenant le sujet dans l'universalité kantienne.

L'auto-transformation de l'espèce reposerait sur la disparition de la limite conceptuelle et de la frontière entre prévention et amélioration. D. Lecourt dénonce le biocatastrophisme ambiant qui voudrait distinguer entre humain et post-humain, ce qui rejetterait tout progrès de la bio-science comme un irrespect de la Nature, de l'Homme ${ }^{14}$. À l'inverse cette dédifférenciation, qu'opèrerait la biotechnologie pour Habermas, n'autorise plus la personne à être dans son propre corps car pouvoir être soi-même postule une identité et une transmission du corps vivant :

«Or, pour que la personne puisse ne faire qu'un avec son corps, il semble qu'il lui soit nécessaire de l'éprouver comme s'inscrivant dans la croissance naturelle comme le prolongement de la vie organique, se régénérant elle-même, dont la personne est issue par sa naissance $"{ }^{15}$.

N'y a-t-il pas dans ce postulat d'une transcendance du corps vivant dont il faudrait préserver l'intégralité naturelle afin d'exercer une pleine subjectivité ?

L'identification au corps vivant, si elle assure une incarnation de la personne dans une filiation naturelle, ne suffit pas pour garantir au sujet une acceptation de son état essentiel ou accidentel. La dépossession de l'origine par le design génétique de son existence est aussi une source de liberté, à défaut de libération, face aux conséquences individuelles, relationnelles, affectives et sociales de la dépendance corporelle du handicap corporel et/ou mental. Faut-il refuser le progrès biotechnologique dès lors qu'il propose, il est vrai dans l'après-coup d'un choix effectué sans le consentement du sujet, des possibilités d'existence et d'action plus grandes que ce que le sujet aurait pu être sans. Que pourrait dire le sujet, comme en témoigne l'affaire Perruche, s'il pouvait connaître qu'il aurait pu être différent? L'interrogation sur l'identité virtuelle par 
rapport à l'identification au corps vivant provient du corps vécu, réfléchi, informé et revendiquant, désormais, une construction biosubjective du soi.

La critique du progrès biotechnologique ne peut plus en rester à l'opposition corps vivant/corps humain. La biotechnologie transforme le rapport du sujet à la nature en faisant de son corps un résultat environnemental. Désormais les parents pourront être responsables de la malformation ou non de leur enfant à naître car ils peuvent ou non connaître le destin génétique de leur enfant : le corps de l'enfant devient un support de valeur, un mode de valorisation et un contrôle actif de la transmission. La définition culturelle du corps peut désormais se transmettre à l'insu du sujet à naître mais au nom du bien sanitaire prédéfini. La connaissance biotechnologique précède le sujet à naître en lui donnant une santé normative sans laquelle les parents estiment qu'il subirait l'inégalité naturelle. Le droit de l'enfant à naître peut-il se fonder sur l'intention d'une bonne santé ? La responsabilité des parents de choisir un corps handicapant malgré la connaissance anténatale peut-il être le choix d'une valeur analogue à celui de la fidélité et l'abstinence qui refuse le préservatif, de la contraception ou de l'avortement?

Le corps est l'objet d'un jugement de valeur, que nous placions notre espoir dans la transcendance de la Nature, dans l'universalité de la Personne ou dans l'intention sanitaire anténatale. Quelle valeur s'imposerait-elle comme meilleure pour tous dans une culture du corps de chacun et de la construction de l'identité personnelle? Nos enfants légitiment l'évidence de la libération du corps en la portant désormais sur le droit à disposer du corps avant et au moment de la mort. S'en étonner est essentiel, mais la biosubjectivité est une mise en culture du corps : le corps n'est plus seulement l'objet culturel du sujet, mais la matière identitaire d'un soi mobile et vivant.

De l'humilité économique à l'humiliation du corps

$\mathrm{Au}$ moment où l'imaginaire contemporain se préoccupe du clonage humain, moins comme possibilité technique réelle que comme représentation d'un avenir entièrement bio-artificiel, les conditions d'existence des corps exclus du jeu économique n'est plus qu'une préoccupation caritative: le Téléthon rapporte davantage que les restos du cœur, le chômage est banalisé, le RMI tend à être remplacé par le RMA, l'insertion de l'autre en moins. Chacun est désormais identifié, contrôlé et pire s'identifie à son corps : comme la carte, le corps est d'identité ${ }^{16}$. Il y a non seulement une évolution historique, mais aussi une typologie de l'action sociale qui, même aujourd'hui, peut constituer une référence pour chaque acteur du travail social. Même si les contraintes du travail social doivent être distinctes du regard psycho-affectif du sujet-acteur de cette action aujourd'hui d'insertion.

Le modèle de la vie de Christ va servir de référence à l'évaluation de la pauvreté. Être pauvre, c'est à la fois être pauper et humilis : c'est-à-dire que le pauvre, doux et affligé, a besoin de Dieu. La pauvreté du Christ est ontologique et existentielle : comme le précise Jean-Louis Goglin : «Le Christ accepte de devenir homme, il en accepte la dimension, lui qui est Dieu, il s'est fait pauvre parce que l'homme est pauvre, et sa mort sur la Croix découle de cette acceptation de la dimension humaine... Sa renonciation aux richesses divines, les seules vraies, l'a donc conduit aux souffrances de sa vie et de sa mort: la pauvreté et la souffrance ne sont pas dissociées ${ }^{17}$.

31 Le mendiant de l'Ancien Régime ne peut être comparé au pauvre moderne. D'une part à cause des conditions économiques qui séparaient réellement les riches et les pauvres, d'autre part à cause de la structure sociale qui n'engageait pas le rapport à la mendicité de la même façon que notre relation moderne à la pauvreté. La misère errante est avant 
tout « l'histoire d'une charité qui puisait son mobile le plus profond dans le sentiment religieux, regardant le pauvre comme un élu de Dieu. Entre le riche et le pauvre s'établissait une relation personnelle de l'ordre d'un échange symbolique, le don du premier contre les prières du second. Leurs rôles sociaux en sortaient confirmés, et le soulagement de la misère de l'un rachetait le salut de l'autre, l'allégeait du poids moral de sa richesse ${ }^{18}$. Cet équilibre symbolique était le moyen de maintenir le système féodal: la religion, qui attribuait à chaque individu une nature comme son destin, participait de ce conservatisme.

Cet équilibre symbolique est conçu non seulement au plan individuel en attribuant à la charité une dimension morale de devoir métaphysique; mais aussi comme un traitement d'ensemble qui apportait une solution institutionnelle dans l'enfermement et l'obligation du travail dans l'espace clos des hôpitaux et hospices. La charité individuelle s'alliait collectivement à une répression sociale organisée. Michel Foucault a montré comment l'édit de création de l'Hôpital général en 1656 précise que cette institution doit: "empêcher la mendicité et l'oisiveté comme sources de tous les désordres ». Ainsi, «puisque la paresse est devenue la forme absolue de la révolte, on contraindra les oisifs au travail, dans le loisir indéfini d'un labeur sans utilité ni profit ${ }^{19}$ : cette association entre paresse et mendicité va donner au travail le projet d'une "transcendance éthique», par laquelle la proscription de l'oisiveté est combattue. La communauté de travail constitue déjà la référence morale par rapport à laquelle les misérables, les gueux seront parqués.

L'enjeu est surtout moral plutôt qu'économique. Ainsi, l'origine de la pauvreté est située à partir de "l'affaiblissement de la discipline et le relâchement des mœurs " plutôt que la rareté des denrées ou le chômage. Tout l'appareil juridique de la répression est au service de l'obligation de travail dans ces institutions et est chargé de châtier et de corriger une certaine "vacance " morale. Le travail est le moyen d'un exercice de réforme, de contrainte morale, d'ascèse, de punition, comme signe d'une attitude du cœur. Delamare écrit, dans son Traité de Police (1738) : « ... la tempérance éloignerait les maladies; l'assiduité au travail, la frugalité, et une sage prévoyance procureraient toujours les choses nécessaires à la vie ; la charité bannissant les vices, la tranquillité publique serait assurée ; l'humilité et la simplicité retrancheraient ce qu'il y a de vain et de dangereux dans les sciences humaines; la bonne foi régnerait dans les sciences et dans les arts... les pauvres seraient enfin secourus volontairement et la mendicité bannie ; il est vrai de dire que la religion seule étant bien observée, toutes les autres parties de la police seraient accomplies ". L'internement est donc un moyen de protection sociale du négatif de l'homme : la pauvreté est perçue comme la négation de l'essence morale de l'homme créé par Dieu.

Et surtout, il s'agit d'évacuer de la scène sociale une pauvreté massive sans avoir le souci de considérer chaque cas individuel : l'enfermement et l'internement sont des moyens de purifier la scène sociale tout en organisant en un lieu clos (à la fois bonne conscience de la société et purification morale) une obligation salvatrice du travail. La finalité de l'internement est la protection de la société. Déjà le traitement collectif de la pauvreté par la mise au travail, même s'il est inutile économiquement, reste anonyme et neutre : «Les pauvres existaient, on les voyait, on ne les connaissait cependant pas : dans le geste individuel de charité envers le mendiant, la misère n'avait pas de visage, faute d'éléments qui en auraient rendu possible une lecture d'ensemble ${ }^{20}$. Le travail est pensé en terme d'occupation car la valeur économique de cette population n'a pas 
été découverte comme elle le sera dans la constitution en classe sociale du prolétariat à partir du début du XIX ${ }^{e}$ siècle. Sans cette individualisation, les thérapies par le travail n'auront pas pour but de réinsérer les individus mais de les maintenir dans une discipline morale et juridique suffisamment sévère pour les contrôler de manière continue. On secourt les pauvres sans distinction.

C'est la pensée sociale du XVIII ${ }^{e}$ siècle (de Montesquieu aux physiocrates et à Rousseau) qui inspire l'aspiration à laïciser la charité. L'inadaptation de la politique de la mendicité exige des transformations sociales. Ainsi, par l'émanation des comités de salut public, un gouvernement représentatif considère la misère comme une priorité de l'action politique. Pour arriver à un système d'assistance publique, il convient d'inscrire les recettes et les dépenses pour l'assistance dans le budget de l'État plutôt que d'entériner l'ancienne charité privée de prescription religieuse. L'assistance est un service national : alors que l'ancienne charité était aléatoire, occasionnelle, arbitraire, la société nouvelle se fait un devoir de protéger les pauvres comme ses propres membres. On reconnaît les droits de l'homme pauvre sur la société et ceux de la société sur lui. Rousseau avait établi le modèle du corps politique en faisant de chaque citoyen un membre (au sens organique) : si bien que l'état de chaque membre est en relation directe avec l'ensemble du corps politique.

Cependant ce processus de laïcisation, au lieu d'établir un traitement égalitaire, va établir une distinction au sein de la pauvreté. Ainsi, une dichotomie est instaurée tout au long du XvIII ${ }^{\mathrm{e}}$ siècle entre le vice et la vertu : le vagabond professionnel est assimilé à un personnage vicieux qui ne souhaite pas se réinsérer dans la société, tandis que le pauvre honteux est la figure du vertueux. Le vagabond est perçu comme un danger, dans la mesure où l'oisiveté est contraire, précise l'article « Oisiveté » de L'Encyclopédie, " aux devoirs de l'homme et du citoyen, dont l'obligation générale est d'être bon à quelque chose, et en particulier de se rendre utile à la société dont il est membre. Rien ne peut dispenser personne de ce devoir, parce qu'il est imposé par la nature ». La différence entre les pauvres et les mendiants maintient l'opposition économique, mais refuse la mendicité assimilée à la fainéantise. Le modèle physiocratique accorde au travail une fonction d'utilité naturelle et de cohérence économique du corps social. Contre le vagabondage, Turgot propose une classification des pauvres afin d'organiser rationnellement les secours : une fois distingués les pauvres recensés dans leur lieu de vie des vagabonds, deux sortes de secours seraient apportés. Les secours gratuits pour les malades hors d'état pour subvenir à leurs besoins, des salaires pour les autres. Se développe ainsi un droit à la subsistance, droit qui ne vaut que pour les citoyens : «L'un des premiers actes de l'Assemblée Nationale constituante, dès le début de l'année 1790, fut en effet la création d'un Comité de mendicité, sous la direction de La Rochefoucauld-Liancourt. Dans le décret de constitution (21 janvier), les députés insistaient sur la nécessité d'organiser un traitement d'ensemble de la misère et sur l'urgence d'une étude des moyens qui pourraient la faire disparaître " ${ }^{21}$.

Conclusion : Le droit de disposer de sa propre mort ou les limites du respect

En privilégiant les soins palliatifs plutôt que l'euthanasie ${ }^{22}$, comme en interdisant le clonage, même thérapeutique, la société française indique les limites du respect: le corps de la personne est unique et chrétien; sa souffrance comme sa douleur feraient partie de l'expérience humaine de la naissance à la mort; il conviendrait de mourir convenablement en respectant la temporalité aménagée par l'accompagnement. Le droit à disposer de son propre corps, du corps d'autrui s'arrêterait à la question de la 
mort, dont l'État disposerait encore en légiférant sur le permis d'inhumer, sur le droit de conserver le corps mort, sur les carrés religieux dans les cimetières municipaux et en refusant au sujet le droit de disposer de sa propre mort. Le corps devrait être respecté en fin de vie, là où l'avortement de confort élimine l'enfant à naître dépisté anormal et là où la médecine réparatrice modifie indéfiniment le corps naturel. Cette résistance de nos valeurs chrétiennes pose la question du respect du mourant.

Le corps malade, les stratégies sont non seulement visibles, malgré les masques, mais se traduisent immédiatement dans et sur le corps du malade. Ainsi faut-il dire ou parler de la vérité au malade, l'éthique du corps soignant doit-elle être tenue au secret médical ? À ne fréquenter que rituellement le malade, le corps médical peut faire du secret une position et une retraite. Mais le soin, surtout s'il ne s'avère plus curatif, engage le personnel soignant précisément dans le domaine du corps personnel: un contrat de confiance peut alors s'établir dès lors que l'apaisement de la douleur, comme si le corps soignant devait répondre à la demande d'un Autre qui ne peut rien faire pour son propre corps. Prends soin de mon corps ou de ma mort, moi qui n'ai pas la force ou la volonté d'en finir ${ }^{23}$. Aussi convient-il de ne pas confondre la douleur physique avec la souffrance morale ${ }^{24}$. L'impuissance de partager la souffrance, alors que la douleur peut et doit être soulagée, place le corps soignant dans une situation de culpabilité : la tentation de s'identifier au malade, en confondant la sphère publique de l'action avec la sphère privée de la compassion, est grande pour le corps soignant. Le soin prend alors du sens, car la souffrance est une demande de sens adressé à Autrui, représenté par le corps soignant, par des malades de plus en plus isolés du milieu familial. Dans une approche pédagogique, «la base de la relation soigné-soignant est bien le contrat de soin, contrat tacite, oral et synallagmatique... ce qui n'est pas négociable ce sont essentiellement les valeurs du soigné... ce qui n'est pas négociable pour le soignant, c'est la clause de conscience ${ }^{25}$. Hors de ce contrat de confiance, le corps soignant pourrait oublier la nécessité d'introduire du sens dans ses actes.

Car prendre soin du corps d'autrui introduit le corps soignant dans une approche tactile : ce qui est livré, à travers le corps objectif, correspond à la chair intime. "Médecins comme infirmières ont de plus en plus tendance à s'éloigner du corps du malade, considérant comme des activités "en plus", voire subalternes, des tâches comme la toilette du malade. Ce contact corporel est pourtant essentiel, pour le malade comme pour le soignant. C'est à partir de moments de ce type que peuvent filtrer des éléments permettant de faire retrouver au malade une situation plus unifiée, permettant une meilleure adhésion à la thérapeutique ${ }^{26}$. Sans cette éthique du toucher, le corps soignant ne trouvera pas son geste propre, restant ainsi dans la logique du corps médical ${ }^{27}$. 


\section{NOTES}

1.. B. ANDRIEU, «Éduquer son corps : une valeur adolescente », dans E. PRAIRAT, B. ANDRIEU eds., Les Valeurs. Savoir et éducation à l'école, Presses Universitaires de Nancy, 2003, p. 129-141.

2.. Selon le titre du dossier de la nouvelle revue le Minotaure, $\mathrm{n}^{\circ} 1$, Printemps 2003,p. 80-105.

3.. David Le BRETON, La Peau et la Trace. Sur les blessures de soi, Paris, Metailié, 2003, p. 13.

4.. B. ANDRIEU, « Choisir son corps », Les Cultes du corps. Éthique et sciences, Paris, L'Harmattan, 1994, p. 261-283.

5.. Dont on peut lire la sténotypie des débats dans le livre préfacé par Simone de BEAUVoIR, Avortement : une loi en procès ; l'affaire de Bobigny, Paris, Idées/Gallimard, 1973, $\mathrm{n}^{\circ} 283$.

6.. B. PRECIADO, «Qu'est-ce que la contra sexualité ? », Manifeste contra-sexuel, Paris, Balland, 2000, p. 21.

7.. Jean-Antonin GITENET, « La boîte de pandore lesboqueer », Hermaphrodite, $\mathrm{n}^{\circ} 8$, Porno(graphie), 2003, p. 100-109.

8.. Marie-Hélène BOURCIER, Queer zones. Politique des identités sexuelles des représentations et des savoirs, Paris, Balland, 2001.

9.. B. ANDRIEU, Un corps à soi. Critique du masochisme, Éditions Euredit, euredit@wanadoo.fr, 2000.

10.. ESTELLE, Deux mille hommes, Paris, Press Pocket, 2003. Raffaëlla ANDERSON, Hard, Paris, Le livre de poche, 2003.

11.. B. ANDRIEU, «L'élimination du handicap », L'Interprétation des gènes. Un exemple de confusion des savoirs, Paris, L'Harmattan, coll. « Le mouvement des savoirs », 2002, p. 137-162.

12.. B. ANDRIEU, « Médecin de soi-même : du mythe à la somatechnie ", Médecin de son corps, Paris, PUF, Préface François Dagognet, 1999, p. 7-11.

13.. J. HABERMAS, L'Avenir de la nature humaine, Paris, Gallimard, 2002.

14.. D. LECOURT, «Bio-catatrophisme et posthumanité », Humain, post humain. La technique et la vie, Paris, PUF, 2003, p. 17-55.

15.. HABERMAS, L'Avenir..., op. cit., p. 131.

16.. Bernard ANDRIEU, « Le corps d'identité », Les Plaisirs de la chair. Une philosophie politique du corps, Paris, Le temps des cerises, 1998, p. 9-27.

17.. Jean-Louis GoGLIN, Les Misérables dans l'Occident médiéval, Paris, Seuil, Points/ Histoire, 1976, p. 28.

18.. Giovanna PROCACCI, Gouverner la misère. La question sociale en France, 1789-1848, Paris, Seuil, 1993, p. 37.

19.. Michel FoucAult, « Le grand renfermement », Histoire de la folie à l'Âge classique [1961], Paris, Gallimard, coll. « Tel », 1972, p. 84.

20.. G. PROCACCI, Gouverner la misère, op. cit., p. 39.

21.. Ibid., p. 65.

22.. Bernard ANDRIEU, « Faire corps : être sujet du corps mourant ", La Nouvelle Philosophie du corps, Toulouse, Éditions Érès, 2002, p. 145-168. 
23.. « Les femmes du dernier acte ", La Mort à Vivre, Autrement, $\mathrm{n}^{\circ}$ 87, février 1987, p. 117-120.

24.. Jérôme PORÉE, « La souffrance comme ouverture à l'autre ", La Philosophie à l'épreuve du mal. Pour une phénoménologie de la souffrance, Paris, Vrin, 1993, p. 267-282.

25.. Louis et Nicole LERY, « Éthique et pluridisciplinarité : contrat de soin, négociation, approche pédagogique », in Actes du séminaire interdisciplinaire d'éthique, Université Bordeaux II-U.F.R de Santé Publique, Éd. M. Le Sommer Pere, février 1992, p. 63-64. 26. Françoise LouX, Traditions et soins d'aujourd'hui, Paris, Inter Éditions, 1990, p. 61. 27. Bibliographie complémentaire : Jole AGRINI, Chiara CRISCIANI, « Charité et assistance dans la civilisation chrétienne médiévale ", dans Histoire de la pensée médicale en Occident, [1993], Paris, Seuil, 1995, p. 151-174. B. ALLEMANDOU, J.-J. LE PENNEC, 60000 pauvres à Bordeaux. La politique d'Aide Sociale sous la Révolution, Bordeaux, Éd. Maison des Sciences de l'Homme d'Aquitaine, 1995, 2 tomes. Michel AUTES, Travail social et pauvreté, Paris, Syros, 1992, 320 p. Colette BEC, Assistance et république. La recherche d'un nouveau contrat social sous la III République, Paris, Éd. de l'Atelier, 1994, 254 p. Yves-Marie BERCE, Croquants et nu-pieds. Les soulèvements paysans en France du XVI ${ }^{e}$ au XIX siècle, [1974], Paris, Folio/Histoire 34, 1991, 303 p. José CUBERO, Histoire du vagabondage du Moyen Âge à nos jours, Imago, 1998, 292 p. François EWALD, Histoire de l'État Providence. Les origines de la solidarité, [1986], Livre de Poche 4222, 1996, 317 p. A. GUESLIN, P. GuILLAME (éd.), De la charité médiévale à la sécurité sociale. Économie de la protection sociale du Moyen Âge à l'époque contemporaine, Éd. Ouvrières, 1992, 249 p. Henri HATZFELD, Du paupérisme à la sécurité sociale, 1850-1940. Essai sur les origines de la sécurité sociale en France, [1971], Presse Universitaires de Nancy, 1989, 344 p. Michel IMBERT, Les Hôpitaux en France [1958], Paris, PUF, coll. «Que sais-je ? », 1994. E. LA ROSA, Santé, précarité et exclusion, Paris, PUF, coll. «Le Sociologue », 1998, 222 p. René LENOIR, Les Exclus. Un français sur dix, Paris, ActuelsSeuil, 1973, 180 p. François-Xavier MERRIEN (éd.), Face à la pauvreté. L'Occident et les pauvres hier et aujourd'hui, Paris, Éditions de l'Atelier, 1994, 269 p. Serge PAUGAM, La Société française et ses pauvres. L'expérience du revenu minimum d'insertion, Paris, PUF, 1993, 317 p. Pierre rosanVALLon, L'État en France de 1789 à nos jours, Paris, Seuil, 1990, 370 p. Lionel STOLERU, Vaincre la pauvreté dans les pays riches, Paris, Champs/Flammarion, 1977, 319 p. Alexandre VEXLIARD, Le Clochard [1957], Paris, Desclée de Brouwer, 1998, 485 p.

\section{RÉSUMÉS}

À l'heure du clonage, de la guerre et de l'incivilité, le respect est aujourd'hui mis en avant comme LA valeur essentielle de la socialité, de la moralité et de la civilité. Mais, derrière cette apparence normative, les pratiques corporelles révèlent combien le malade, le pauvre, le chômeur et le mourant ne profitent pas de ce respect. Comment fonder un respect de la personne à partir d'un respect réel des corps?

With the clonage, the war and the incivility, the respect is today the moral value of our socialibility, morality and civility. But, behind this normative appearance, the body practices 
discover the sick, the poor, the unemployment, and the dying man with a lack of respect. How to found a respect for the person from a real respect of bodies?

\section{AUTEUR}

\section{BERNARD ANDRIEU}

Bernard Andrieu est Maître de conférences habilité en Épistémologie à l'IUFM de Lorraine, historien et philosophe du corps ; bernard.andrieu@wanadoo.fr 